\title{
Tadalafil improves lean mass and endothelial function in nonobese men with mild ED/LUTS: in vivo and in vitro characterization
}

\author{
Antonio Aversa ${ }^{1}$ - Simona Fittipaldi ${ }^{2} \cdot$ Davide Francomano $^{3}$ - Viviana M. Bimonte ${ }^{4}$. \\ Emanuela A. Greco ${ }^{3,5}$ - Clara Crescioli ${ }^{4}$ Luigi Di Luigi ${ }^{4} \cdot$ Andrea Lenzi $^{3}$. \\ Silvia Migliaccio ${ }^{4}$
}

Received: 27 July 2016 / Accepted: 10 December 2016

(C) Springer Science+Business Media New York 2017

\begin{abstract}
Purpose Phosphodiesterase type-5 inhibitor administration in diabetic men with erectile dysfunction (ED) is associated with reduced waist circumference. We evaluated potential effects of daily tadalafil administration on body composition and investigated its possible mechanism(s) of action in $\mathrm{C}_{2} \mathrm{C}_{12}$ skeletal muscle cells in vitro.

Methods Forty-three men on stable caloric intake (mean age $48.5 \pm 7$; BMI $25.5 \pm 0.9 \mathrm{~kg} / \mathrm{m}^{2}$ ) complaining mild ED and/or low urinary tract symptoms (LUTS) were randomly assigned to receive tadalafil (TAD) $5 \mathrm{mg} /$ daily (once-aday=OAD-TAD; $n=23$ ) or $20 \mathrm{mg}$ on-demand (on-demand= OD-TAD; $n=20$ ) for 2 months. Primary outcomes were variations of body composition measured by Dual-energy X-ray absorptiometry; secondary outcomes were ED/LUTS questionnaire scores along with hormone (testosterone, estradiol, insulin) and endothelial function (Endopat2000) variations.

Results OAD-TAD increased abdominal lean mass $(p<$ $0.01)$ that returned to baseline after 2 months withdrawal.

Antonio Aversa

aversa@unicz.it

1 Department of Experimental and Clinical Medicine, Magna Græcia University, Catanzaro, Italy

2 IRCCS SDN, Napoli, Italy

3 Department of Experimental Medicine, Section of Medical Pathophysiology, Endocrinology and Nutrition, "Sapienza" University of Rome, Rome, Italy

4 Department of Movement, Human and Health Sciences, Section of Health Sciences, "Foro Italico" University of Rome, Rome, Italy

5 LiSa Laboratory, Policlinico Catania, University of Catania, Catania, Italy
\end{abstract}

LUTS scores improved $(p<0.01)$ in OD-TAD while ED scores improved $(p<0.01)$ in both groups. We found significant improvements in endothelial function $(p<0.05)$ that directly correlated with serum insulin $(p<0.01$; $r=0.3641)$ and inversely correlated with estradiol levels $(p<0.01 ; r=0.3655)$ even when corrected for potential confounders. Exposure of $\mathrm{C}_{2} \mathrm{C}_{12}$ cells upon increasing tadalafil concentrations $\left(10^{-7}\right.$ to $\left.10^{-6} \mathrm{M}\right)$ increased total androgen receptor mRNA and protein expression as well as myogenin protein expression after 24 and $72 \mathrm{~h}$ (2.8 \pm 0.4 -fold and $1.4 \pm 0.02$-fold vs. control, respectively, $p<0.05)$.

Conclusions Daily tadalafil improved lean mass content in non-obese men probably via enhanced insulin secretion, estradiol reduction, and improvement of endothelial function in vivo. The in vitro increased myogenin and androgen receptor protein expression in skeletal muscle cells suggests a translational action of phosphodiesterase type-5 on this receptor.

Keywords Testosterone $\cdot$ Estradiol $\cdot$ Phosphodiesterase type-5 $\cdot$ Erectile Dysfunction $\cdot$ Androgen receptor $\cdot$ Skeletal muscle $\cdot$ Fat mass $\cdot$ Lower Urinary Tract Symptoms

\section{Introduction}

Phosphodiesterases (PDEs) are a family of enzymes with different selectivity for cyclic nucleotides, cAMP, and cGMP, [1] that play several regulatory roles such as platelet aggregation, T-cell activation, and smooth cell relaxation and vascular tone $[2,3]$. In addition, the NO-cGMP signaling pathway seems to play an important role in the regulation of muscle metabolism [4] and insulin action [5]. 
$\mathrm{PDE}_{5}$ inhibitors $\left(\mathrm{PDE}_{5} \mathrm{i}\right)$ are selective blockers of the cGMP-hydrolyzing enzyme. In men with erectile dysfunction (ED) chronic PDE 5 i improves insulin secretion, [6] endothelial function, [7] and in vitro it regulates aromatase (ARO) expression both in adipocyte [8] and osteoblast cell models. [9] Interestingly, data from experimental rabbit model of high fat diet-induced metabolic syndrome (MetS) confirms that tadalafil (TAD) administration not only after long-term (12 weeks), but even after a short-term exposure ( 1 week), in vivo and in vitro, was able to restore a correct pre-adipocyte commitment, through a positive effect on insulin sensitivity, and to upregulate, via PKG activation, the genes related to mitochondrial biogenesis and brown adipogenesis, including $\mathrm{UCP}_{1}$ [10]. Studies in men with type-2 diabetes $\left(\mathrm{T}_{2} \mathrm{D}\right)$ confirmed that chronic sildenafil administration was able to reduce epicardial adipose tissue and in a murine diabetic model regulated visceral adiposity by shifting adipose tissue cell composition toward a less inflamed profile [11]. No data on possible effects of $\mathrm{PDE}_{5}$ inhibitors on lean mass in humans have been described yet.

We aimed to investigate the potential effects of daily TAD treatment on lean mass, hormonal, and endothelial function in men with mild ED and/or LUTS. We additionally used $\mathrm{C}_{2} \mathrm{C}_{12}$ skeletal muscle cell line to evaluate the in vitro effect of TAD during myogenic differentiation by the analysis of skeletal muscle-specific differentiationdependent markers.

\section{Materials and methods}

\section{Study design}

This was an open label, parallel arm design study aimed to determine the potential effects of TAD assumption on body composition. Forty three male subjects ( $\geq 18$ years-BMI $\geq$ $18.5 \leq 25 \mathrm{~kg} / \mathrm{mg}^{2}$ ) affected by mild ED and/or LUTS were enrolled. They were randomly allocated to receive either TAD $5 \mathrm{mg} /$ daily (OAD-TAD; $n=23$ ) or TAD $20 \mathrm{mg}$ ondemand (OD-TAD; $n=20$ ) for 2 months. Three scheduled visits were planned: baseline, 2-months after each respective treatment, and 2 months follow-up, with following evaluations: general physical examination, anthropometric parameters [body weight (BW), height, BMI, and waist circumference (WC)]; body composition [fat mass (FM), lean mass (FFM)], endothelial function, fasting serum sample, International Index of Erectile Function-5 items (IIEF-5), and International Prostate Symptom Score (IPSS) questionnaires.

Primary outcomes were variations from baseline of body composition; secondary outcomes were variations from baseline of the IIEF-5, IPSS, total testosterone (TT), 17 $\beta$ estradiol (E), T/E ratios, fasting insulin levels, endothelial function (TBo). The clinical study was carried out in an in- office setting at Sapienza University of Rome and each patient signed a detailed informed consent (Study ID 2369, committee approval \#166/12); ClinicalTrials.gov (NCT02554045). To clarify the potential cellular and molecular mechanisms on body composition, in vitro experiments were performed to further highlight intracellular events triggered by TAD exposure in skeletal muscle cells. Thus, $\mathrm{C}_{2} \mathrm{C}_{12}$ cell line was used to investigate the effects of TAD on myogenic differentiation. Given that testosterone (T)-androgen receptor (AR) signaling pathway plays an important role in early myogenesis [12, 13] by increasing AR expression during $\mathrm{C}_{2} \mathrm{C}_{12}$ differentiation [14] and that TAD increases $\mathrm{AR}$ expression and $\mathrm{T}$ release in human osteoblasts, we evaluated effects on AR expression during early stages of muscle cells differentiation.

\section{Exclusion criteria}

IIEF5 $<17$ (patients with severe ED), IPSS $>7$ (patients with severe LUTS), any concomitant treatment during the prior 3 months, lifestyle, diet or physical exercise changes attended within the 16 weeks of study duration; patients potentially trying to become father; significant hepatic, respiratory, hematological or renal disease; history of drugs or alcohol abuse; history or presence of any cancer; any other reason that the investigator feels precludes patients safety.

\section{Dual-energy X-ray absorptiometry (DEXA) assessment}

Body composition was evaluated by DEXA (DEXAHologic QDR-1000), according to previously published procedures [15]. We modified calculations for android abdominal section, by using the area included between the line joining the last inferior ribs and the line joining the superior iliac spines. Long-term precision error was $0.54 \%$ (phantom); short-term precision error was $1.2 \%$ for the lumbar spine and 2\% for the femoral neck. Fat Free Mass (FFM) and Fat Mass (FM) was expressed in grams per square centimeter (g). DEXA soft tissue measurements variation coefficient was FFM: $1.067 \%$ and FM: $1.748 \%$.

\section{Endothelial function}

Endothelial function was investigated by peripheral arterial tonometry (PAT; EndoPAT2000, Itamar Medical), according to previously published procedures [16]. Instead of the natural logarithm of PAT ratio, we evaluated the average amplitude of PAT signal in the post-occlusion period (test signal, T) compared with that in the baseline (B) signal before occlusion, utilizing the same time intervals as for reactive hyperemia index $(\mathrm{RHI})$ calculation, but not indexed to controlateral arm (referred to as T/Bo ratio), as previously published [17]. 


\section{Laboratory analyses}

TT and E were measured by chemiluminescence microparticle immunoassay (CMIA, Architect System, Abbott Laboratories, IL, USA), with a detection limit of $0.28 \mathrm{nmol} / \mathrm{L}$ and $10 \mathrm{pg} / \mathrm{mL}$, respectively (intra-assay and interassay coefficients of variation for TT: 2.1 and $3.6 \%$ at $10.08 \mathrm{nmol} / \mathrm{L}$ ), with reference values $2.8-11 \mathrm{ng} / \mathrm{mL}(\mathrm{T})$ and $25-107 \mathrm{pg} / \mathrm{mL}$ $\left(\mathrm{E}_{2}\right)$. Insulin levels were analyzed by immunometric assay based on chemiluminescence using an automated clinical chemistry analyzer (Immulite 2000, Diagnostic Product Corp., Los Angeles, CA, USA) [18].

\section{Cell culture and treatments}

$\mathrm{C}_{2} \mathrm{C}_{12}$ skeletal muscle cells (CRL-1772; American Type Culture Collection, Manassas, VA, USA) were cultured in Dulbecco's modified Eagle medium without phenol red (DMEM; Euroclone, Milan, Italy) supplemented with 10\% heat-inactivated fetal bovine serum (Invitrogen, Carlsbad, CA, USA) and $1 \%$ penicillin and streptomycin (SigmaAldrich, St. Louis, MO, USA) under an atmosphere of 5\% $\mathrm{CO}_{2}$ in humidified air at $37^{\circ} \mathrm{C}$. In all experiments, cells were seeded at the density of $10^{4} \mathrm{cells} / \mathrm{cm}^{2}$ and allowed to grow until $80 \%$ confluence. To induce myogenic differentiation, fetal bovine serum content was lowered to $2 \%$. TAD (kindly supplied by ELI Lilly ICOS Corporation) was added after the induction of myogenic differentiation. Cells were harvested for molecular analysis after 12, 24, 48, and $72 \mathrm{~h}$ of differentiation.

\section{Protein extraction and immunoblot analysis}

Following treatments, cells were washed with PBS and lysed in fresh ice-cold RIPA buffer (Sigma-Aldrich) supplemented before use with protease and phosphatase inhibitor cocktails (Roche, Indianapolis, IN, USA). Lysates were precleared by centrifugation and protein content was measured using Pierce ${ }^{\circledR}$ BCA protein assay kit (Thermo Fisher Scientific, Waltham, MA, USA). Samples were boiled for $5 \mathrm{~min}$; then an equal amount of protein extracts $(15-30 \mu \mathrm{g})$ was resolved in SDS-polyacrylamide gels (10-14\%) and transferred to polyvinylidene difluoride (PVDF) membranes (Amersham Biosciences, Piscataway, NJ, USA). Membranes were saturated by incubation at room temperature for $1 \mathrm{~h}$ with $5 \%(\mathrm{w} / \mathrm{v})$ non-fat dry milk in $20 \mathrm{mM}$ Tris- $\mathrm{HCl}, 150 \mathrm{mM} \mathrm{NaCl}, 0.1 \%$ Tween 20 , and incubated with specific first antibodies (1:1000) overnight at $4{ }^{\circ} \mathrm{C}$. Antibodies against class I myosin (MyoD), Myogenin, and myosin heavy chain (MyHC) (H300) were from Santa Cruz Biotechnology (Dallas, Texas, USA); AR, CYP19, and $\beta$-actin antibodies from Cell Signaling Technology (Danvers, MA, USA); $\beta$-actin and GAPDH antibodies from
Sigma-Aldrich. Immunoreactive protein bands were detected by incubation for $1 \mathrm{~h}$ with horseradish peroxidaseconjugated secondary goat anti-rabbit $(1: 10000$, Merck Millipore, Billeric, MA, USA) or goat anti-mouse (1:10000, Sigma-Aldrich) in blocking solution at room temperature, visualized by enhanced chemiluminescence (Amersham Biosciences), then bands were obtained on the ImageQuant LAS 4000 (GEHC).

\section{Immunofluorescence and myogenic differentiation assays}

Cells were washed twice with cold PBS, fixed with $4 \%$ formaldehyde, permeabilized with $0.2 \%$ Triton X-100 in PBS and fixed in blocking solution $(6 \mathrm{ng} / \mathrm{ml} \mathrm{IgG}$ from goat serum in PBS) for $30 \mathrm{~min}$. Cells were incubated with anti-MyHC antibody (1:300, Santa Cruz Biotechnology) overnight at $4{ }^{\circ} \mathrm{C}$. Alexa Fluor 488-conjugated anti-rabbit $\operatorname{IgG}(\mathrm{H}+\mathrm{L})$ was used as secondary antibody (1:500, Thermo Fisher Scientific). After counterstaining with 4',6-Diamidino-2-phenylindole dihydrochloride (DAPI; $500 \mathrm{ng} / \mathrm{ml}$ ), slides were mounted with Prolong Gold Antifade Reagent (Invitrogen).

Myogenic differentiation was evaluated by calculating differentiation and fusion indexes representing the proportion of nuclei that were localized within MyHC-positive myotubes and the formation of multinucleated myotubes, respectively. Fluorescent images of random fields were captured with $20 \times$ magnification. For each experimental condition, total number of nuclei and number of nuclei incorporated into the myotubes were counted. The differentiation index was calculated as the percentage of MHCpositive cells above total and fusion index was determined by the percentage of nuclei incorporated into myotubes (defined as containing at least two nuclei) relative to the total number of nuclei.

\section{Measurement of mRNA levels by quantitative RT-PCR}

RNA was extracted using the TRI reagent (Sigma) and subjected to DNase digestion (Thermo Fisher Scientific). Real-time quantitative PCR was conducted using One-step SYBR GreenER (Thermo Fisher Scientific) in a 7500 Real-time PCR System (Applied Biosystems). Specific primers were as follows: $\mathrm{Fw}$ Ar (5'-TACCAGCTCACCAAGCTCCT-3'); Rv AR (5'-GATGGGCTTGA CTTTCCCAG-3'); Fw Cyp19 (5'-AACCCCATGCAGTATAATGTCAC-3'); Rv Cyp19 (5'-AGGACCTGGTATTGAAGACGAG-3'); Fw Cyclophilin A (5'-GTCAACCCC ACCGTGTTCTT-3'); Rv Cyclophilin A (5'-CTGCTGT CTTTGGGACCTTGT-3'). Each analysis was performed in triplicate sessions. Relative expression levels were calculated using the comparative cycle threshold $(\Delta \Delta \mathrm{Ct})$ method using Cyclophilin A as internal control. 


\section{ELISA assay}

After 24 and $72 \mathrm{~h}$ of differentiation in the presence or absence of TAD, media were removed and centrifuged at $500 \mathrm{~g}$ for 10 min at $4{ }^{\circ} \mathrm{C}$, and supernatants stored at $-80{ }^{\circ} \mathrm{C}$ until used for assays. $T$ was measured by sensitive ELISA kit (Enzo Life Sciences, Inc., Plymouth Meeting, PA, USA) assay. Data from ELISA assay in the supernatants were normalized for number of viable cells at the end of each experiment and expressed in $\mathrm{pg} / \mathrm{ml}$. The sensitivity of T kit is $2.6 \mathrm{pg} / \mathrm{ml}$ with a range of detection between 3.9 and $1000 \mathrm{pg} / \mathrm{ml}$.

\section{Statistical analysis}

For the clinical study, results are reported as mean \pm standard error when normally distributed, and as median (quartiles) when non-parametric. Data were analyzed using $t$-tests for single comparisons, analysis of covariance for comparisons at specific time points, using baseline score as a covariate. Multiple stepwise regression analysis was performed to determine associations between serum endothelial function, $\mathrm{E}$ and insulin concentration after adjusting for potential confounders such as age, cigarette smoking, and drug/supplemental facts assumption. A $p$-value $<0.05$ was considered statistically significant. Statistical analysis was performed using the computer statistical package SPSS 11.0 (SPSS Inc., Chicago, IL, USA).

For the in vitro study, all results represent the means \pm $\mathrm{SD}$ of at least three independent experiments, and $p$-values were calculated using the unpaired $t$-test.

\section{Results}

\section{TAD improves body composition in vivo}

After 2 months of both TAD treatments, no significant changes in total $\mathrm{BW}$ or overall FFM/FM were found (Table 1). Interestingly, 2 months OAD-TAD induced an increase in FFM (expressed as percentages) both at the trunk (Fig. 1a; $p<0.01$ ) and android sections (Fig. 1b; $p<$ 0.001 ) that was lost after 2 months withdrawal, whereas a similar trend in appendicular FFM was also found (data not shown). Accordingly, OAD-TAD produced a trend to reduction in $\mathrm{WC}(-2.5 \mathrm{~cm}$, Table 1$)$, with significant reduction of abdominal FM (\%) only at the android section that returned to baseline after 2 months from withdrawal (Fig. 1c; $p<0.01$ ). IIEF-5 questionnaire also showed a significant improvement for both treatment groups (Table 1; $p<0.01$, while IPPS scores improved significantly in OAD-TAD only $(p<0.01)$. OAD-TAD induced a significant drop in E levels $(p<0.001)$ while no modifications in circulating TT levels were found, determining significant reduction of $\mathrm{T} / \mathrm{E}$ ratio (Table $1 ; p<0.01$ ) that returned to baseline after withdrawal.

Endothelial function (T/Bo) showed significant improvement after 2 months OAD-TAD (Fig. 2a; $p<0.01$ ) that returned at baseline after withdrawal. Improvements in endothelial function showed a direct correlation with insulin (Fig. 2b; $p<0.01, r: 0.3641$ ) and an inverse correlation with E serum levels (Fig. 2c; $p<0.01, r$ : 0.3655), respectively. No differences in body composition, endothelial function,

Table 1 Clinical characteristics at baseline of the ITT population, after 2-months treatment with daily TAD 5 mg and on-demand TAD 20 mg, and after 2-months from withdrawal

\begin{tabular}{|c|c|c|c|c|c|c|c|c|}
\hline \multicolumn{5}{|c|}{ OAD-TAD $(n=23)$} & \multicolumn{4}{|c|}{ OD-TAD $(n=20)$} \\
\hline \multicolumn{5}{|c|}{ Mean Age (ys): $48 \pm 7$} & \multicolumn{4}{|c|}{ Mean Age (ys): $49 \pm 7$} \\
\hline & Baseline & $\begin{array}{l}2 \text { months } \\
\text { treatment }\end{array}$ & $\begin{array}{l}2 \text { months } \\
\text { withdrawal }\end{array}$ & $p$ & Baseline & $\begin{array}{l}2 \text { months } \\
\text { treatment }\end{array}$ & $\begin{array}{l}2 \text { months } \\
\text { withdrawal }\end{array}$ & $p$ \\
\hline Weight $(\mathrm{Kg})$ & $77.8 \pm 16.8$ & $81.0 \pm 14.9$ & $77.1 \pm 15.5$ & $\mathrm{~ns}$ & $75.4 \pm 13.9$ & $76.3 \pm 12.6$ & $77.1 \pm 13.1$ & ns \\
\hline BMI & $25.5 \pm 0.9$ & $25.9 \pm 1.0$ & $25.4 \pm 1.1$ & ns & $26.2 \pm 0.8$ & $26.1 \pm 1.1$ & $26.4 \pm 1.0$ & ns \\
\hline Waist $(\mathrm{cm})$ & $97.5 \pm 3.3$ & $95.0 \pm 2.1$ & $97.2 \pm 3.1$ & ns & $98.8 \pm 4.4$ & $99.8 \pm 3.1$ & $99.3 \pm 3.9$ & ns \\
\hline Lean total $(\%)$ & $76.2 \pm 4.1$ & $76.5 \pm 4.3$ & $76.3 \pm 3.2$ & ns & $76.1 \pm 4.3$ & $76.2 \pm 4.1$ & $76.0 \pm 3.9$ & ns \\
\hline Fat total $(\%)$ & $21.6 \pm 3.7$ & $21.1 \pm 3.6$ & $21.3 \pm 2.8$ & ns & $21.7 \pm 3.1$ & $21.6 \pm 3.4$ & $21.8 \pm 3.3$ & ns \\
\hline Total T (ng/mL) & $5.76 \pm 1.5$ & $5.46 \pm 1.71$ & $5.65 \pm 1.45$ & ns & $5.13 \pm 1.1$ & $4.92 \pm 1.87$ & $5.45 \pm 1.63$ & $\mathrm{~ns}$ \\
\hline $\mathrm{E}(\mathrm{pg} / \mathrm{mL})$ & $35.2 \pm 9.22$ & $20.75 \pm 4.09 * *$ & $34.1 \pm 8.32$ & $* * 0.001$ & $38.23 \pm 6.76$ & $32.56 \pm 8.34$ & $39.6 \pm 5.98$ & ns \\
\hline Total T/E & $0.175 \pm 0.07$ & $0.30 \pm 0.1 *$ & $0.180 \pm 0.09$ & $* 0.01$ & $0.156 \pm 0.09$ & $0.169 \pm 0.8$ & $0.146 \pm 0.09$ & ns \\
\hline SHBG (nmol/L) & $39.4 \pm 3.1$ & $40.1 \pm 2.8$ & $38.6 \pm 2.9$ & $\mathrm{~ns}$ & $41.3 \pm 3.8$ & $43.2 \pm 4.7$ & $39.8 \pm 3.7$ & ns \\
\hline Insulin (mIU/l) & $5.9 \pm 0.5$ & $9.1 \pm 0.3^{*}$ & $6.1 \pm 0.4$ & $* 0.01$ & $5.4 \pm 0.3$ & $5.9 \pm 0.7$ & $5.3 \pm 0.5$ & ns \\
\hline IIEF 5 & $19 \pm 1.5$ & $24 \pm 0.5^{*}$ & $22 \pm 1.5$ & $* 0.01$ & $18 \pm 1.0$ & $25 \pm 1.5^{*}$ & $20 \pm 1.5$ & $* 0.01$ \\
\hline IPSS & $7.5 \pm 0.5$ & $3.1 \pm 0.3^{*}$ & $7.3 \pm 0.4$ & $* 0.01$ & $7.3 \pm 0.6$ & $7.9 \pm 0.5$ & $8.3 \pm 0.4$ & ns \\
\hline
\end{tabular}

$B M I$ body mass index $\left(\mathrm{kg} / \mathrm{m}^{2}\right), E$ 17ß-Estradiol, SHBG Sex Hormone Binding Globulin, IIEF5 International Index of Erectile Function-5 items, IPSS International Prostate Symptoms Score 
Fig. 1 Differences after daily TAD treatment in lean and FM (gr=grams) from baseline. Daily TAD $5 \mathrm{mg}$ produced a) significant variation in lean trunk fat ( $p<0.01,2-$ months vs. baseline) that returned at baseline levels after 2 months from withdrawal; b) significant variation android trunk lean mass (gr) $(p<0.001-2$-months vs. baseline) that returned at baseline levels after 2 months from withdrawal. Also,

c) a significant variation in android trunk FM (gr) $(p<0.01$ -2-months vs. baseline) that returned at baseline value after 2 months from withdrawal was found
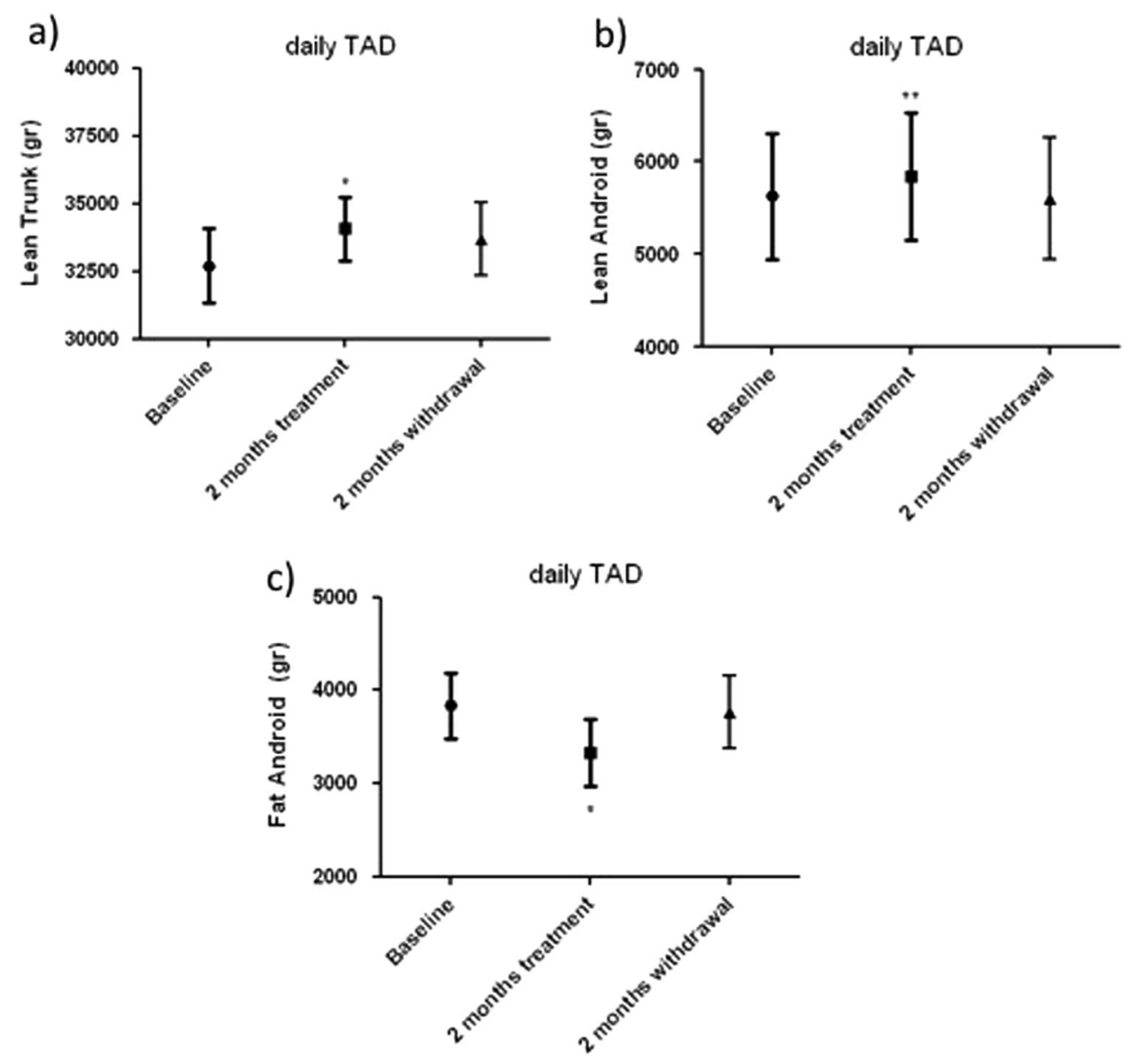

and T/E ratios were found after OD-TAD treatment. Side effects were those commonly reported with TAD administration (headache and myalgia) did not differ between the two groups and did not determine drug discontinuation (data not shown).

\section{TAD modulates myogenic differentiation in $\mathrm{C}_{2} \mathrm{C}_{12}$ skeletal muscle cells in vitro}

For in vitro experiments, TAD concentrations were chosen $\left(10^{-7}\right.$ to $\left.10^{-6} \mathrm{M}\right)$ based on previous studies $[8,9,19]$, indicating these concentrations as likely equivalent to the doses used for in vivo treatment. To evaluate potential effect of TAD on initiation of differentiation program, cells were treated with increasing concentrations of TAD and key myogenic differentiation markers, such as MyoD, myogenin, and MyHC, were analyzed after 24 and $72 \mathrm{~h}$ exposure by western blot analysis.

TAD $\left(10^{-7}\right.$ to $\left.10^{-6} \mathrm{M}\right)$ induced a $3.3 \pm 0.7$-fold and a 3.9 \pm 0.9 -fold increase in MyoD protein expression after $24 \mathrm{~h}$ exposure as compared with untreated cells, while TAD $\left(10^{-6} \mathrm{M}\right)$ induced a myogenin protein expression increase of
$2.8 \pm 0.4$-fold and $1.4 \pm 0.02$-fold vs. control cells after 24 and $72 \mathrm{~h}$, respectively. The same concentrations of TAD $\left(10^{-7}\right.$ to $\left.10^{-6} \mathrm{M}\right)$ induced $1.4 \pm 0.07$-fold and $1.6 \pm 0.2$-fold increase in MyHC protein expression, respectively, after 24 h (Fig. 3a, b).

Fusion index for myotubes formation in the differentiated cells increased after $72 \mathrm{~h}$ of about $30 \%$ from the onset of differentiation process upon $10^{-6} \mathrm{M}$ TAD exposure as compared with untreated cells (Fig. 3d, e). Preliminary experiments, performed to demonstrate lack of cytotoxicity effects and to assess cell viability, by trypan blue exclusion assay and MTS assay, did not demonstrate differences between treated and untreated cells (data not shown).

\section{TAD increases AR expression}

As depicted in Fig. 4a, RT-qPCR analysis revealed a $1.3 \pm$ 0.08 -fold increase of AR mRNA content in cells treated with $10^{-6} \mathrm{M}$ TAD for $12 \mathrm{~h}(p<0.05)$ reaching a $1.5 \pm 0.08$ fold increase after $24 \mathrm{~h}(p<0.05)$ as compared with untreated cells. Increase was lost after $48 \mathrm{~h}$. Western blot analysis further supported an increase in $A R$ protein 
Fig. 2 Treatment with daily TAD treatment produced a significant improvement of a) endothelial function (T/Bo, $p<0.05$; 2-months vs. baseline) that returned at baseline levels after 2 months from withdrawal. Endothelial function (T/Bo) directly correlated with b) insulin $(p<0.01 ; r=0.3641)$ and inversely correlated with c) E serum levels $(p<0.01$; $r=0.3655)$, respectively
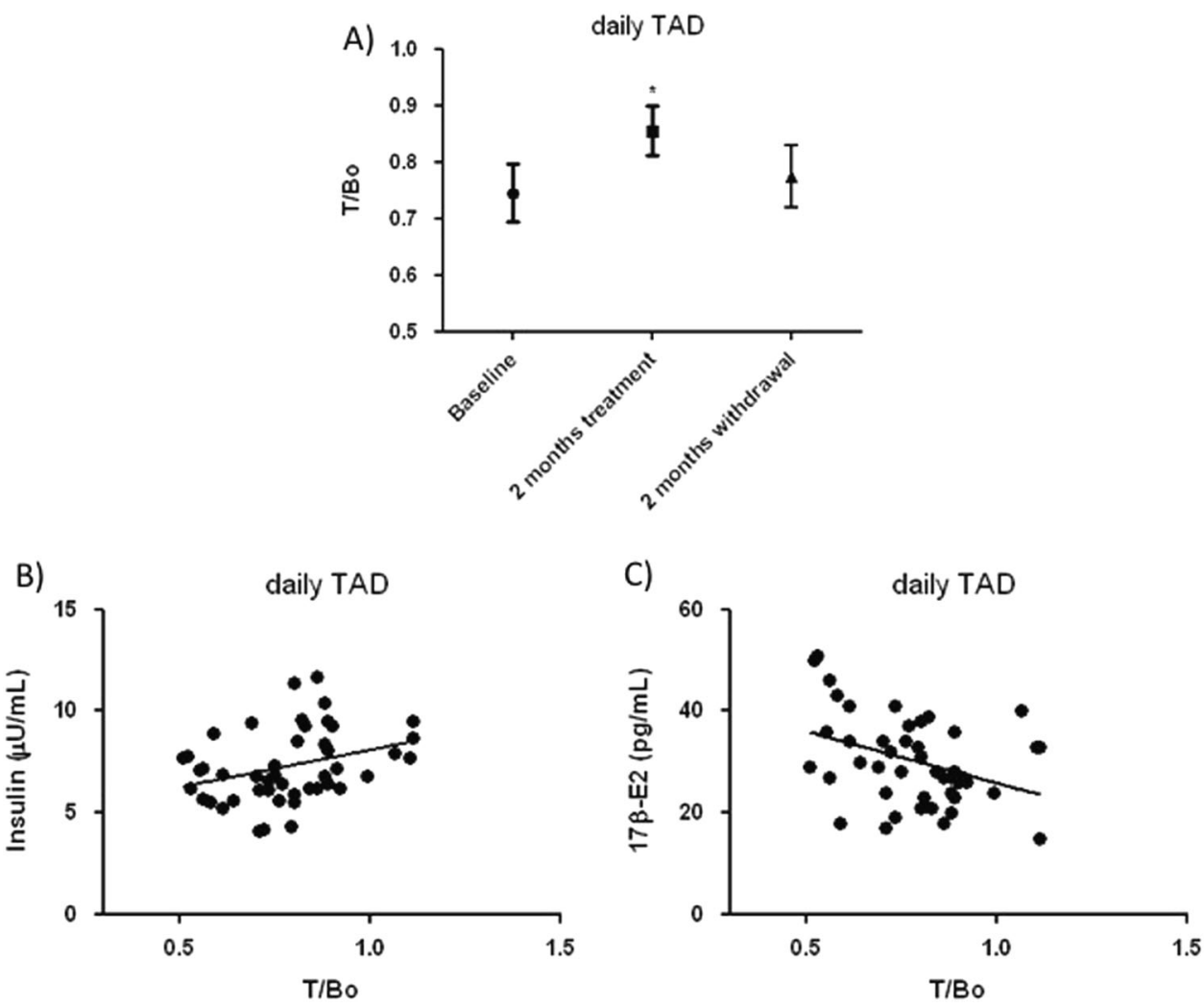

expression $(2.0 \pm 0.3$-fold $)$ in TAD-treated vs. untreated cells $(p<0.05)$ (Fig. 4b). Since a rapid regulation of Cyp19 (ARO) mRNA and protein level was previously observed in TAD-treated human adypocytes, eight potential modulations were evaluated in muscle cells as well. TAD $\left(10^{-6} \mathrm{M}\right)$ induced a 1.4 \pm 0.07-fold increase of Cyp19 mRNA expression in the cells $12 \mathrm{~h}$ after the differentiation induction ( $p<0.05$, data not shown), but the effect was blunted after $24 \mathrm{~h}$. Moreover, western blot analysis showed a $2.5 \pm$ 0.7-fold increase of Cyp19 protein levels in TAD-treated cells at $24 \mathrm{~h}$ and a return to control level after $48 \mathrm{~h}(p<$ 0.05 , data not shown).

In accordance with an upregulation of AR expression, a relevant increase of $\mathrm{T}$ concentrations in the supernatant of $10^{-6} \mathrm{M}$ TAD-treated cells $(2.3 \pm 0.5$-fold compared with untreated cells, $p<0.05$ ) was found after 24-h treatment, that was also blunted after $48 \mathrm{~h}$ (data not shown). Unfortunately, it was not possible to detect $\mathrm{E}$ levels in $\mathrm{C}_{2} \mathrm{C}_{12}$ supernatant cells, since values resulted too low as with commercial high sensitive ELISA kits available (data not shown).

Since $\mathrm{T}$ regulates $\mathrm{PDE}_{5}$ expression and responsiveness to TAD in human corpus cavernosum, [20] the expression level of this enzyme was evaluated during $\mathrm{C}_{2} \mathrm{C}_{12}$ muscle cells differentiation with or without TAD. $\mathrm{PDE}_{5} \mathrm{a}$ mRNA level increased after 48-h differentiation into myotubes (1.5 \pm 0.02 vs. undifferentiated cells, data not shown), confirming previous studies in human primary skeletal muscle cells [21]. By contrast, TAD did not affect PDE $_{5}$ a mRNA expression in the same experimental conditions.

\section{Discussion}

This is the first demonstration that shows that short-term daily TAD administration improves body composition in men by concomitant increases in FFM and decreases in FM with a tendency to decrease in WC. These latter results are in line with studies in vivo in the rabbit model, showing beneficial effects of TAD on visceral adipose tissue preadipocyte phenotype and amelioration of MetS features, that were corroborated by further demonstrating an in vitro upregulation of expression of genes related to brown/beige commitment, and mitochondrial fusion. [10] In our young healthy subject affected by mild ED/LUTS, we found interesting modifications in hormonal milieu, i.e., higher T/ $\mathrm{E}$ ratio and increased insulin production, along with multivariate analysis showing significant association with insulin, E plasma levels, and endothelial function. This 

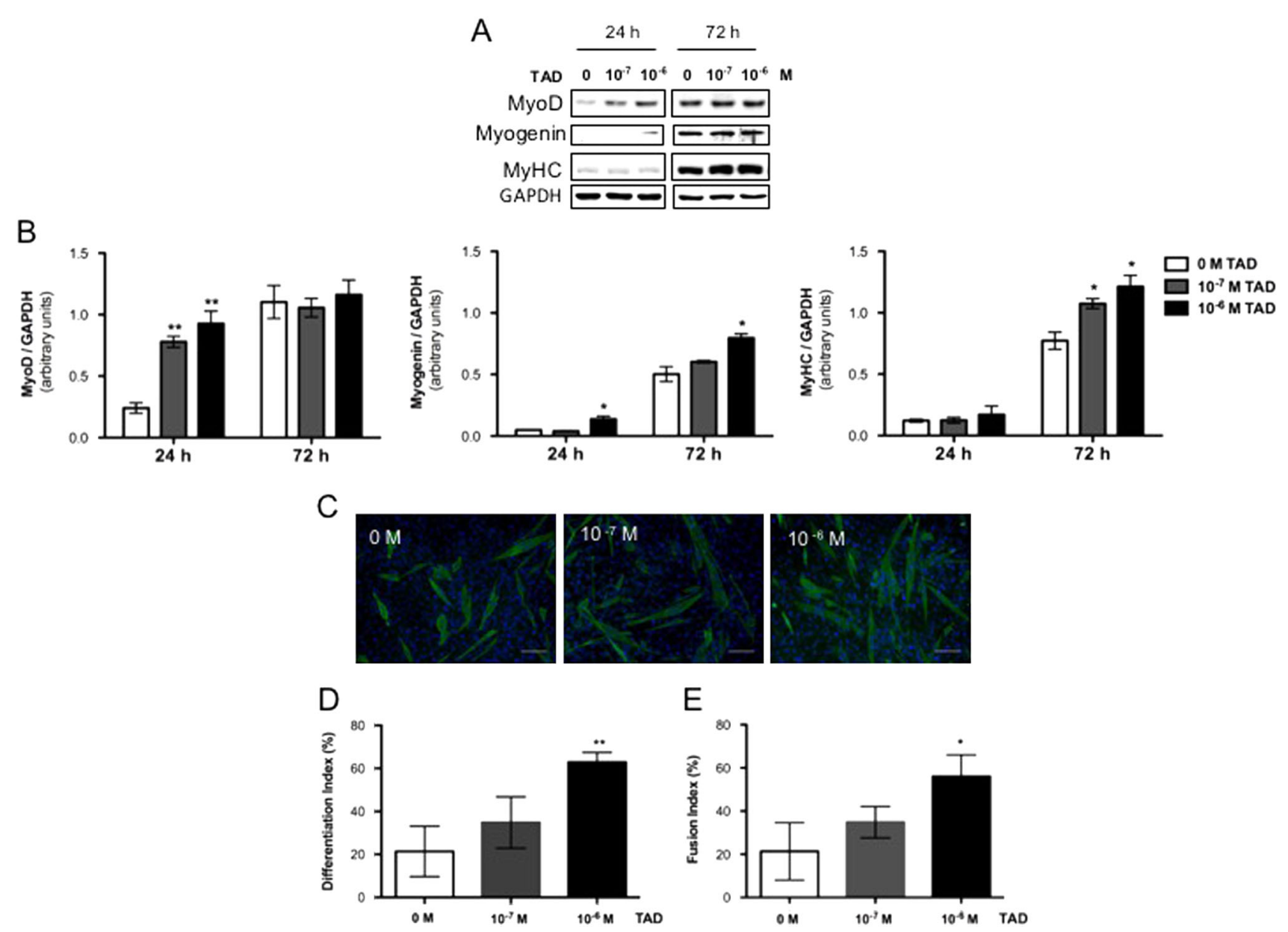

Fig. 3 TAD effect on muscle cells differentiation and myotube formation in vitro. $\mathrm{C}_{2} \mathrm{C}_{12}$ muscle cells were induced to differentiate in the presence or absence of different concentrations of TAD $\left(10^{-7}\right.$ to $\left.10^{-6} \mathrm{M}\right)$. Cell lysates were collected after 24 and $72 \mathrm{~h}$ of differentiation and analyzed by Western blotting. a) Representative Western blot analysis of MyoD, Myogenin and MyHC protein contents b) Histograms represent mean values of MyoD, Myogenyn and MyHC protein levels ratio normalized over the GAPDH protein level c) Representative image of immunofluorescence staining for MyHC after $72 \mathrm{~h}$ of differentiation. Differentiation d) and fusion indexes e) in cells treated with or without TAD $\left(10^{-7}\right.$ to $\left.10^{-6} \mathrm{M}\right)$ were calculated as reported in materials and methods. Results are presented as mean \pm SD $(n=3)$ from three different experiments. Scale bars $=75 \mu \mathrm{m}$; ** $p \leq$ 0.01 and $* p \leq 0.05$ vs. untreated cells result was relevant, since obtained with no changes either in lifestyle and/or physical activity during the study.

The efficacy of long-term treatment with $\mathrm{PDE}_{5} \mathrm{i}$ awaits demonstration in human metabolic diseases, i.e., $\mathrm{T}_{2} \mathrm{D}$ and MetS. Preclinical data suggest that cGMP signaling affects energy balance, insulin resistance, and glucose metabolism, and might be related to vasodilation (resulting in increased perfusion and substrate delivery to metabolically active skeletal muscle) - through direct effects on peripheral glucose handling [22, 23]. Chronic treatment with sildenafil in a mouse model of diet-induced insulin resistance caused a significant improvement in energy expenditure and insulin sensitivity [24]. In animal models, chronic sildenafil improved cardiac performance [25] and reduced interstitial cardiac fibrosis [26]. In men with $\mathrm{T}_{2} \mathrm{D}$, Fiore et al. demonstrated that daily sildenafil improved $\mathrm{WC}$ and epicardial fat, and demonstrated that this occurred by regulation of miR22 release and SIRT1.[11] Also Ramirez et al. demonstrated that chronic sildenafil administration in overweight men with pre-diabetes enhanced insulin sensitivity and improved markers of endothelial function [27]. Hill et al. demonstrated that TAD administration (10 mg on alternate days for 3 weeks in men with MetS) was associated with improved pancreatic $\beta$-cell function, as assessed by oral glucose tolerance testing, when compared with placebo [28]. Accordingly, acute TAD administration had beneficial effects on peripheral microcirculation and glucose uptake also in insulin-resistant diabetic women [29]. Recent discoveries support the evidence that endothelial dysfunction may be the cause of insulin resistance and $\mathrm{T}_{2} \mathrm{D}$, due to a decrease in NO levels, reducing cGMP production, and impairing muscle glucose uptake [30]. Due to TAD action 

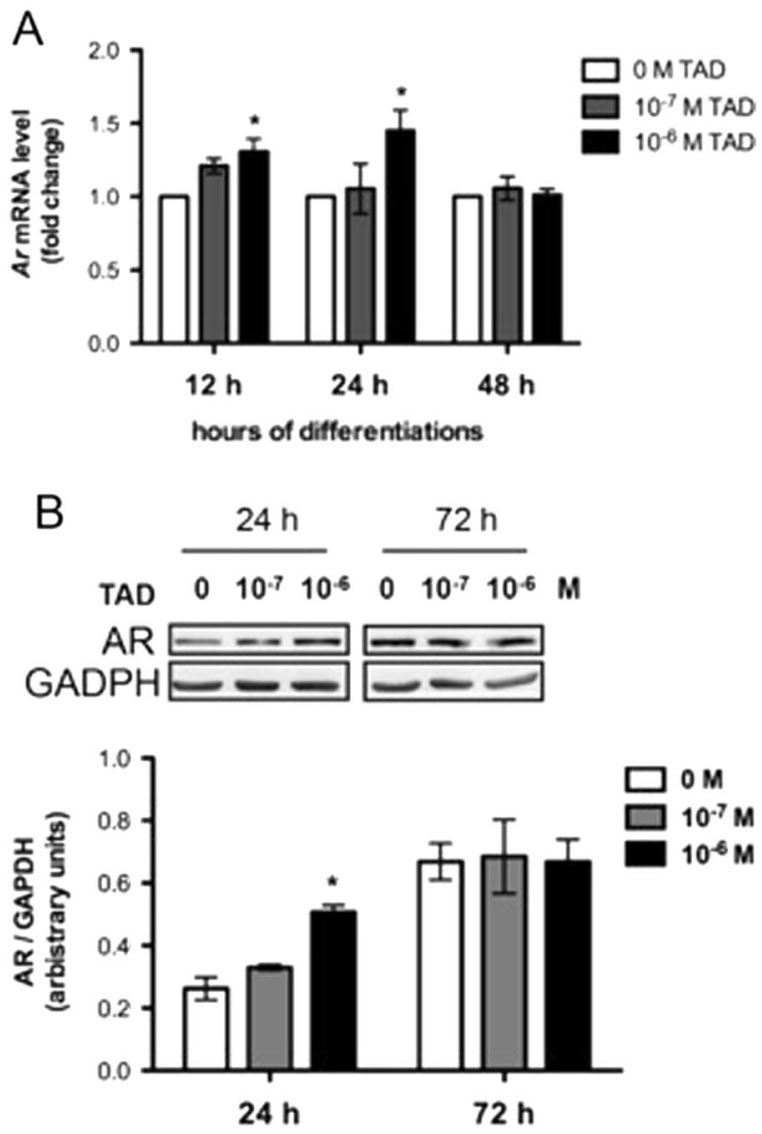

Fig. 4 Effects of TAD $\left(10^{-7}\right.$ to $\left.10^{-6} \mathrm{M}\right)$ on a) AR mRNA expression levels after 12, 24, and $48 \mathrm{~h}$ the onset of differentiation; b) AR protein expression after 24 and $72 \mathrm{~h}$ of differentiation. $* p \leq 0.05$ vs. untreated cells

on insulin and on glucose metabolism in $\mathrm{C}_{2} \mathrm{C}_{12}$ cells, [12] we further investigated possible acceleration of myogenic differentiation and an increased AR expression in these cells. The underlying mechanisms by which T/AR signalling regulates metabolic homeostasis in men are complex, but evidences derived from various $\mathrm{AR}$ knockout mouse models reveal tissue-specific AR signalling that are involved in the regulation of metabolism [31]. MyoD and Myogenin regulate the program of skeletal muscle differentiation: in fact, MyoD plays a crucial role in cells commitment to myogenesis program, while myogenin takes part in the execution of differentiation program [32]. Indeed, we found a relevant increase of MyoD accumulation after $24 \mathrm{~h}$ of differentiation. It is known that the muscle-regulatory transcription factor myogenin appears at a later stage leading to the commitment of differentiation; [33] interestingly, TAD-treated cells showed an earlier transcription after $24 \mathrm{~h}$ differentiation when compared with the control ones. MyHC is part of sarcomeric structure of skeletal muscle and occurs at the end terminal differentiation into functional myotube [34]. When the cells were treated with TAD, it was observed an increase of $\mathrm{MyHC}$ expression and myotube fusion index after $72 \mathrm{~h}$ from the onset of differentiation. Since clinical study showed a clear increase in T/E ratio, [35] a more favorable androgen milieu is hypothesized in vivo that could activate AR transduction cascade involved in myogenesis. This pathway may determine a more selective modulation of $\mathrm{AR}$ and acceleration of the differentiation process in skeletal muscle cells in vitro, [36] suggesting that this event might be one of the potential mechanism leading to the improvement of FFM in our clinical study. The possible relationship between TAD use, AR pathway physiology, muscle cell differentiation, and hormones adaptation [37] should be evaluated in healthy athletes abusing with TAD [38] and androgenic anabolic steroids.

We recognize several limitations of this study. Despite any changes in lifestyle or caloric intake were permitted to patients throughout the entire study duration, the lack of a placebo arm limits the interpretation of our results. It is important to recognize that DEXA may not accurately assess body composition when compared with CT-scans, but mostly in markedly obese subjects. No evaluation of inflammation markers was provided. In addition, although in vitro results suggest a possible role of AR signalling pathway in mediating TAD effects on skeletal muscle cell differentiation, further studies are warranted to elucidate the translational mechanisms involved in TAD effects on skeletal muscle myogenesis.

In conclusion, we demonstrated for the first time that TAD exerts modulatory effects on body composition in vivo in metabolically healthy men and on skeletal muscle cell differentiation in vitro. As expected, mild ED symptoms improved in both groups, while LUTS amelioration was evident during daily administration only. The hormonal and microcirculation variations induced by TAD in clinical subjects accompanied by in vitro muscle cell line differentiation suggest a translational action of $\mathrm{PDE}_{5} \mathrm{i}$ on skeletal muscle possibly mediated via AR.

Acknowledgements The authors thank Dr. Andrea D'Anselmo for revising the English language.

Funding This study was funded by MIUR Grants (grant number 2015XCR88M_008 to Prof. Antonio Aversa and 052013 to Prof. Silvia Migliaccio) and Ministry of Health Grant (grant number 00829 to Prof. Andrea Lenzi).

\section{Compliance with ethical standards}

Competing interests The authors declare that they have no competing interest.

Ethical approval All procedures performed in studies involving human participants were in accordance with the ethical standards of the institutional and/or national research committee and with the 1964 Helsinki declaration and its later amendments or comparable ethical standards (NCT Identifier: NCT02554045). 
Informed consent Informed consent was obtained from all individual participants included in the study.

Animal Rights This article does not contain any studies with animals performed by any of the authors.

\section{References}

1. S.H. Soderling, J.A. Beavo, Regulation of cAMP and cGMP signalling: new phosphodiesterases and new functions. Curr. Opin. Cell. Biol. 12, 174-179 (2000)

2. N.T. Dickinson, E.K. Jang, R.J. Haslam, Activation of cGMPstimulated phosphodiesterase by nitroprusside limits cAMP accumulation in human platelets: effects on platelet aggregation. Biochem. J. 323, 371-377 (1997)

3. K. Omori, J. Kotera, Overview of PDEs and their regulation. Circ. Res. 100, 309-327 (2007)

4. M.E. Young, B. Leighton, Evidence for altered sensitivity of the nitric oxide/cGMP signalling cascade in insulin-resistant skeletal muscle. Biochem. J. 329, 73-79 (1998)

5. S. Collins, T.L. Martin, R.S.J. Surwit, J. Robidoux, Genetic vulnerability to diet-induced obesity in the C57BL/6J mouse: physiological and molecular characteristics. Physiol. Behav. 81, 243-248 (2004)

6. A. Aversa, E. Greco, R. Bruzziches, M. Pili, G. Rosano, G. Spera, Relationship between chronic tadalafil administration and improvement of endothelial function in men with erectile dysfunction: a pilot study. Int. J. Impot. Res. 19, 200-207 (2007)

7. D. Santi, E. Giannetta, A.M. Isidori, C. Vitale, A. Aversa, M. Simoni, Effects of chronic use of phosphodiesterase inhibitors on endothelial markers in type 2 diabetes mellitus: a meta-analysis. Eur. J. Endocrinol. 172, R103-114 (2015)

8. A. Aversa, M. Caprio, A. Antelmi et al. Exposure to phosphodiesterase type 5 inhibitors stimulates aromatase expression in human adipocytes in vitro. J. Sex. Med. 8, 696-704 (2011)

9. A. Aversa, S. Fittipaldi, V.M. Bimonte et al. Tadalafil modulates aromatase activity and androgen receptor expression in a human osteoblastic cell in vitro model. J. Endocrinol. Invest. 39, 199-205 (2016)

10. E. Maneschi, I. Cellai, A. Aversa et al. Tadalafil reduces visceral adipose tissue accumulation by promoting preadipocytes differentiation towards a metabolically healthy phenotype: studies in rabbits. Mol. Cell. Endocrinol. 424, 50-70 (2016)

11. D. Fiore, D. Gianfrilli, E. Giannetta et al. PDE5 inhibition ameliorates visceral adiposity targeting the miR-22/SIRT1 pathway: evidence from the CECSID Trial. J. Clin. Endocrinol. Metab. 101, 1525-1534 (2016)

12. K.D. Lee, Androgen receptor enhances myogenin expression and accelerates differentiation. Biochem. Biophys. Res. Commun. 294, 408-413 (2002)

13. S. Sabatini, P. Sgrò, G. Duranti, R. Ceci, L. Di Luigi, Tadalafil alters energy metabolism in $\mathrm{C} 2 \mathrm{C} 12$ skeletal muscle cells. Acta. Biochim. Pol. 58, 237-242 (2011)

14. F. Wannenes, M. Caprio, L. Gatta, A. Fabbri, S. Bonini, C. Moretti, Androgen receptor expression during $\mathrm{C} 2 \mathrm{C} 12$ skeletal muscle cell line differentiation. Mol Cell Endocrinol. 24(292), 11-19 (2008)

15. M.P. Rothney, Y. Xia, W.K. Wacker et al. Precision of a new tool to measure visceral adipose tissue (VAT) using dualenergy XRay absorptiometry (DXA). Obesity (Silver Spring). 21, E134-E136 (2013)

16. A. Aversa, D. Francomano, R. Bruzziches et al. The application of digital pulse amplitude tonometry to the diagnostic investigation of endothelial dysfunction in men with erectile dysfunction. Andrologia 43, 9-15 (2011)
17. A. Aversa, C. Letizia, D. Francomano, R. Bruzziches, M. Natali, A. Lenzi, A spontaneous, double-blind, double-dummy cross-over study on the effects of daily vardenafil on arterial stiffness in patients with vasculogenic erectile dysfunction. Int. J. Cardiol. 160, 187-191 (2012)

18. G. Antonini, A. Clemenzi, E. Bucci et al. Hypogonadism in DM1 and its relationship to erectile dysfunction. J. Neurol. 258, 1247-1253 (2011)

19. C. Crescioli, N. Sturli, M. Sottili, P. Bonini, A. Lenzi, L. Di Luigi, Insulin-like effect of the phosphodiesterase type 5 inhibitor tadalafil onto male human skeletal muscle cells. J. Endocrinol. Invest. 36, 1020-6 (2013)

20. A. Morelli, S. Filippi, R. Mancina et al. Androgens regulate phosphodiesterase type 5 expression and functional activity in corpora cavernosa. Endocrinology. 145, 2253-2263 (2004)

21. S. Engeli, A.L. Birkenfeld, P.M. Badin, V. Bourlier, K. Louche, $\mathrm{N}$. Viguerie et al. Natriuretic peptides enhance the oxidative capacity of human skeletal muscle. J. Clin. Invest. 122, 4675-46759 (2012)

22. H. Duplain, R. Burcelin, C. Sartori et al. Insulin resistance, hyperlipidemia, and hypertension in mice lacking endothelial nitric oxide synthase. Circulation. 104, 342-345 (2001)

23. M.E. Young, B. Leighton, Evidence for altered sensitivity of the nitric oxide/cGMP signalling cascade in insulin-resistant skeletal muscle. Biochem. J. 329(Pt 1), 73-79 (1998)

24. J.E. Ayala, D.P. Bracy, B.M. Julien et al. Chronic treatment with sildenafil improves energy balance and insulin action in high fatfed conscious mice. Diabetes. 56, 1025-1033 (2007)

25. F.N. Salloum, A. Abbate, A. Das et al. Sildenafil (Viagra) attenuates ischemic cardiomyopathy and improves left ventricular function in mice. Am. J. Physiol. Heart. Circ. Physiol. 294, H1398-H1406 (2008)

26. N.G. Pérez, M.R. Piaggio, I.L. Ennis et al. Phosphodiesterase 5A inhibition induces $\mathrm{Na}+\mathrm{H}+$ exchanger blockade and protection against myocardial infarction. Hypertension 49, 1095-1103 (2007)

27. C.E. Ramirez, H. Nian, C. Yu, J.L. Gamboa, J.M. Luther, N.J. Brown, C.A. Shibao, Treatment with Sildenafil Improves Insulin Sensitivity in Prediabetes: A Randomized, Controlled Trial. J Clin Endocrinol Metab. 100, 4533-4540 (2015)

28. K.D. Hill, A.W. Eckhauser, A. Marney, N.J. Brown, Phosphodiesterase 5 inhibition improves beta-cell function in metabolic syndrome. Diabetes Care 32, 857-859 (2009)

29. P.A. Jansson, G. Murdolo, L. Sjögren, B. Nyström, M. Sjöstrand, L. Strindberg et al. Tadalafil increases muscle capillary recruitment and forearm glucose uptake in women with type 2 diabetes. Diabetologia 53, 2205-2208 (2010)

30. J. Tooke, The association between insulin resistance and endotheliopathy. Diabetes Obes. Metab. 1(Suppl. 1), S17-S22 (1999)

31. I.C. Yu, H.Y. Lin, J.D. Sparks, S. Yeh, C. Chang, Androgen receptor roles in insulin resistance and obesity in males: the linkage of androgen-deprivation therapy to metabolic syndrome. Diabetes 63, 3180-3188 (2014)

32. S.J. Tapscott, The circuitry of a master switch: Myo-d and the regulation of skeletal muscle gene transcription. Development 132, 2685-2695 (2005)

33. V. Andrés, K. Walsh, Myogenin expression, cell cycle withdrawal, and phenotypic differentiation are temporally separable events that precede cell fusion upon myogenesis. J. Cell. Biol. Feb. 132, 657-666 (1996)

34. J. Henningsen, K.T. Rigbolt, B. Blagoev, B.K. Pedersen, I. Kratchmarova, Dynamics of the skeletal muscle secretome during myoblast differentiation. Mol. Cell. Proteomics 9, 2482-2496 (2010)

35. E.A. Greco, M. Pili, R. Bruzziches, G. Corona, G. Spera, A. Aversa, Testosterone: estradiol ratio changes associated with long- 
term tadalafil administration: a pilot study. J. Sex. Med. 3, 716-722 (2006)

36. Y. Kanno, R. Ota, K. Someya, T. Kusakabe, K. Kato, Y. Inouye, Selective androgen receptor modulator, YK11, regulates myogenic differentiation of $\mathrm{C} 2 \mathrm{C} 12$ myoblasts by follistatin expression. Biol. Pharm. Bull. 36, 1460-1465 (2013)

37. L. Di Luigi, C. Baldari, P. Sgrò et al. The type 5 phosphodiesterase's inhibitor tadalafil influences salivary cortisol, testosterone and dehydroepiandrosterone sulfate response to maximal exercise in healthy man. J. Clin. Endocrinol. Metab. 93, 3510-3514 (2008)

38. L. Di Luigi, C. Baldari, F. Pigozzi et al. The long-acting phosphodiesterase inhibitor tadalafil does not influence athletes' VO2max, aerobic, and anaerobic thresholds in normoxia. Int. J. Sports Med. 29, 110-115 (2008) 